University of Texas at El Paso

ScholarWorks@UTEP

3-2009

\title{
Empirical Formulas for Economic Fluctuations: Towards A New Justification
}

Tanja Magoc

The University of Texas at El Paso, tmagoc@utep.edu

Vladik Kreinovich

The University of Texas at El Paso, vladik@utep.edu

Follow this and additional works at: https://scholarworks.utep.edu/cs_techrep

Part of the Computer Engineering Commons

Comments:

Technical Report: UTEP-CS-09-04a

Published in the Proceedings of the 28th North American Fuzzy Information Processing Society Annual Conference NAFIPS'09, Cincinnati, Ohio, June 14-17, 2009.

\section{Recommended Citation}

Magoc, Tanja and Kreinovich, Vladik, "Empirical Formulas for Economic Fluctuations: Towards A New Justification" (2009). Departmental Technical Reports (CS). 30.

https://scholarworks.utep.edu/cs_techrep/30

This Article is brought to you for free and open access by the Computer Science at ScholarWorks@UTEP. It has been accepted for inclusion in Departmental Technical Reports (CS) by an authorized administrator of ScholarWorks@UTEP.For more information, please contact Iweber@utep.edu. 


\title{
Empirical Formulas for Economic Fluctuations: Towards A New Justification
}

\author{
Tanja Magoč and Vladik Kreinovich \\ Department of Computer Science \\ University of Texas at El Paso \\ $500 \mathrm{~W}$. University \\ El Paso, Texas 89968, USA \\ Emails: t.magoc@gmail.com,vladik@utep.edu
}

\begin{abstract}
To avoid crisis developments, it is important to make financial decisions based on the models which correct predict the probabilities of large-scale economic fluctuations. At present, however, most financial decisions are based on Gaussian randomwalk models, models which are known to underestimate the probability of such fluctuations. There exist better empirical models for describing these probabilities, but economists are reluctant to use them since these empirical models lack convincing theoretical explanations. To enhance financial stability and avoid crisis situations, it is therefore important to provide theoretical justification for these (more) accurate empirical models. Such a justification is provided in this paper.
\end{abstract}

\section{IntRoduction to the PRoblem}

\section{A. It Is Important to Take into Account Economic Fluctuations}

It is known that stock prices (and other related economic indices) fluctuate in an unpredictable ("random") way. Usually, these fluctuations are small, but once in a while, they become large.

Large negative fluctuations bring havoc to the economy and finance, lead to crisis situations. It is therefore important to correctly take such fluctuations into account. In particular, it is extremely important to correctly predict the probability of large fluctuations.

\section{B. Gaussian Random Walk Model: A Brief Description}

The quantitative study of stock prices can be traced back to a pioneering $\mathrm{PhD}$ dissertation of $\mathrm{L}$. Bachelier [2] who has shown that for any fixed time quantum, the probabilities of stock price fluctuations of different size can be well described by a Gaussian random walk (what physicists call Brownian motion).

In the Gaussian random walk model, fluctuations of different sizes $x$ are normally distributed, with the exponentially decreasing probability density function

$$
\rho(x)=\frac{1}{\sqrt{2 \pi} \cdot \sigma} \cdot \exp \left(-\frac{x^{2}}{2 \sigma^{2}}\right) .
$$

The random walk model indeed describes small fluctuations reasonably well.

\section{Gaussian Random Walk Model: Limitations}

While the Gaussian random walk model well describes the probabilities of small fluctuations, this model drastically underestimates the probabilities of large fluctuations. For example,

- in the normal distribution, fluctuations larger than $6 \sigma$ have a negligible probability $\approx 10^{-8}$, while

- in real economic systems, even larger fluctuations occur every decade (and even more frequently).

When we underestimate the probability of large fluctuations, we thus underestimate risk - and become unprepared when large fluctuations occur.

\section{Mandelbrot's Fractal Model: A Brief Description}

In the 1960s, Benoit Mandelbrot, the author of fractal theory, empirically studies the fluctuations and showed that larger-scale fluctuations follow the power-law distribution, with the probability density function

$$
\rho(x)=A \cdot x^{-\alpha},
$$

for $\alpha \approx 2.7$.

This empirical result, together with similar empirical discovery of power laws in other application areas, has led to the formulation of fractal theory; see, e.g., [10].

\section{E. Mandelbrot's Fractal Model: Limitations}

Further empirical analysis has shown that while Mandelbrot's model correctly predicts medium-size fluctuations, it drastically overestimates the probability of large-scale fluctuations; see, e.g., [19].

Indeed, in this model, the probability $P\left(x>x_{0}\right)$ that a fluctuation is larger than a certain value $x_{0}$ decreases as

$$
P\left(x>x_{0}\right) \sim \frac{1}{x_{0}^{1.7}}
$$

In other words, for two different thresholds $x_{0}<X_{0}$, we have

$$
\frac{P\left(x>x_{0}\right)}{P\left(x>X_{0}\right)} \approx\left(\frac{X_{0}}{x_{0}}\right)^{1.7} .
$$

In the stock market, daily fluctuations of $\approx x_{0}=1 \%$ are normal, with probability $P \approx 1$. Thus, the crisis-size 
fluctuations larger than $X_{0}=30 \%$ have to occur with the probability

$$
P\left(x>X_{0}\right) \approx \frac{1}{30^{1.7}} \approx 0.003
$$

Thus, we should expect crises every 1/0.003 days, i.e., every year - and in reality, such large-scale crises are much rarer.

\section{F. Empirical Analysis of Economic Fluctuations: Econo- physics}

Since the 1990s, physicists have performed an empirical analysis of economic fluctuations - by using statistical techniques that have been developed for the analysis of fluctuations in physical systems. This application of physical techniques to economics is known as econophysics; see, e.g., [3], [4], [5], [11], [12], [16].

In particular, this empirical analysis has shown that the probability density function corresponding to large economic fluctuations has the form

$$
\rho(x)=A \cdot x^{-4},
$$

i.e., the form (2) with $\alpha \approx 4$; see, e.g., [6], [19]. The same empirical law, with the same paramater $\alpha \approx 4$, has been observed in different stock markets; see, e.g., [20].

In this model, the probability $P\left(x>x_{0}\right)$ that a fluctuation is larger than a certain value $x_{0}$ decreases according to the cubic law:

$$
P\left(x>x_{0}\right) \sim \frac{1}{x_{0}^{3}} .
$$

\section{G. Econophysics Is Not Yet Widely Used in Financial Engi-} neering: Why

The cubic law provides a much better prediction of large economic fluctuations than Gaussian or Mandelbrot's models. However, in the practical financial engineering applications, this law is rarely used.

According to H. E. Stanley, the founder of econophysics, one of the main reasons why this law is not used is because it is empirical, it lacks a clear economic justification [18]. Without a good theoretical explanation, economists are reluctant to rely on this law being valid in the future as well - and to make serious decisions based on this law.

\section{H. Need for a Theoretical Explanation}

As we have just mentioned, in the existing financial decisions, economists use Gaussian random walk models which are much less accurate than the empirically more accurate econophysics models. The reason for this use is that the econophysics models are purely empirical, without a good intuitive explanation. As a result of this practice, the existing financial instruments underestimate the probability of largescale crisis-type fluctuations.

To make financial systems more reliable and less vulnerable to large-scale crisis-style fluctuations, it is therefore important to overcome the economists' reluctance, and to provide a theoretical explanation for the empirically observed power laws. This need is emphasized, e.g., in [18].

\section{What Is Known and What We Do In This Paper}

There exist several theoretical explanations for the empirical cubic law; see, e.g., [7]. These explanations are based on the deep mathematical analysis of complex systems. The complex mathematical nature of these explanations makes them not very convincing for economists.

The main objective of this paper is to provide simpler - and hopefully more convincing - explanations for the cubic law.

\section{First Part: General Justification of the Power LAW}

\section{A. Main Objective of This Section}

The cubic law, in its form (6), is a particular example of the general power law (2). It is therefore reasonable to start with explaining why we have a power law. After that, in the following sections, we will move further to justify the exact value of the corresponding scaling parameter $\alpha$.

\section{B. Analysis of the Problem: A Practice-Oriented Temporal Reformulation of the Probabilities}

Ideally, we should be able to predict when the fluctuations will reach a given size $x_{0}$. In reality, as we have mentioned, economic fluctuations are random (unpredictable). As a result, we cannot predict the exact moment of time when fluctuations reach the threshold $x_{0}$. Instead, we can only predict the average time $t$ before such a fluctuation occurs.

From this viewpoint, we would like to find the dependence $t\left(x_{0}\right)$ of this average time on the size of the fluctuation.

This dependence is naturally related to the probabilities. Indeed, the probability density function $\rho(x)$ means that the probability of a fluctuation of size $x_{0}$ is equal to $\rho\left(x_{0}\right)$. (To be more precise, it is equal to $\rho\left(x_{0}\right) \cdot h$, where $h$ is the corresponding discretization step).

The probability that the fluctuation of this size occurs within a single time quantum $\Delta t$ is equal to $\rho\left(x_{0}\right) \cdot h$. Thus, the expected number of such fluctuations during a single time quantum is $\rho\left(x_{0}\right) \cdot h$. During the time period $t$, we have $N \stackrel{\text { def }}{=} \frac{t}{\Delta t}$ time quanta. The expected number of fluctuations of size $x_{0}$ during this time period is therefore equal to

$$
N \cdot\left(\rho\left(x_{0}\right) \cdot h\right)=\frac{t}{\Delta t} \cdot\left(\rho\left(x_{0}\right) \cdot h\right) .
$$

The average time $t\left(x_{0}\right)$ until such a fluctuation occurs can be estimated as the time $t$ for which this expected number of fluctuations becomes close to 1 :

$$
\frac{t\left(x_{0}\right)}{\Delta t} \cdot\left(\rho\left(x_{0}\right) \cdot h\right) \approx 1,
$$

hence

$$
t\left(x_{0}\right) \approx \frac{\Delta t}{\rho\left(x_{0}\right) \cdot h} .
$$

Thus, once we find the dependence $t(x)$, we will be able to find the desired probability density function $\rho(x)$ as

$$
\rho(x) \approx \frac{\Delta t}{t(x) \cdot h}=\frac{\text { const }}{t(x)},
$$

where const $\stackrel{\text { def }}{=} \frac{\Delta t}{h}$. 


\section{Scale Invariance: A Natural Requirement}

We want to describe a general dependence $t(x)$ of the average time $t$ during which the fluctuation of a given size occurs on the size $x$ of this fluctuation.

When describing this dependence, one should take into account that the numerical value of the fluctuation size $x$ depends on the choice of a measuring unit for describing fluctuations. In principle, different units can be chosen. For example, when the European countries changed from their original currencies to Euros, all the stock prices at local stock markets were accordingly re-scaled. In general, if instead of the original unit, we use a new unit which is $\lambda$ times smaller, then the fluctuation whose size in the original unit is $x$ has the value $x^{\prime}=\lambda \cdot x$ in the new units.

It is reasonable to require that the expression describing dependence $t(x)$ should not depend on the choice of the unit. One needs to be careful, however, when formulating this natural requirement. Namely, we cannot simply assume that for the same numerical value $x$, the time is the same no matter which units we use. If we use a smaller unit than before, then

- a fluctuation whose size is one new unit is smaller than the fluctuation whose size is one original unit - and thus,

- the time to reach the 1 new unit size fluctuation should be smaller than the time to reach the 1 old unit size fluctuation.

So, to make a proper formalization, we must take into account that if we re-scale the units in which we measure fluctuations, we must accordingly change the units for time.

If we use the new unit for the fluctuation size, then instead of the numerical value $x$, we get a new numerical value $x^{\prime}=$ $\lambda \cdot x$. Thus, instead of the original time $t(x)$, we get a new time $t\left(x^{\prime}\right)=t(\lambda \cdot x)$. We require that this new time is actually the same time as $t(x)$, but expressed in different time units. If we denote the ratio of the corresponding time units by $r(\lambda)$, then we arrive at the formula

$$
t(\lambda \cdot x)=r(\lambda) \cdot t(x) .
$$

Thus, we arrive at the following requirement: for every $\lambda>$ 0 , there exists a value $r(\lambda)$ for which, for all $x$ and for all $\lambda$, we have

$$
t(\lambda \cdot x)=r(\lambda) \cdot t(x) .
$$

\section{Scale-Invariance Implies Power Law}

It is known that every continuous function $t(x)$ satisfying the above property has the form $t(x)=r \cdot x^{\alpha}$ for some $\alpha$; see, e.g., [1], Section 3.1.1, or [13]. (This result was first proven in [15].)

\section{E. Proof}

For differentiable functions $t(x)$, the result about power functions is easy to prove. Indeed, if we differentiate both sides of (13) by $\lambda$ and take $\lambda=1$, we get

$$
x \cdot \frac{d t}{d x}=\alpha \cdot t,
$$

where $\alpha \stackrel{\text { def }}{=} r(1)$. By moving all the terms containing $t$ into one side and all the terms containing $x$ to the other side, we conclude that

$$
\frac{d t}{t}=\alpha \cdot \frac{d x}{x} .
$$

Integrating both sides, we get

$$
\ln (t)=\alpha \cdot \ln (x)+c,
$$

hence

$$
t=e^{\alpha \cdot \ln (x)+c}=e^{c} \cdot\left(e^{\ln (x)}\right)^{\alpha}=C \cdot x^{\alpha}
$$

for $C=e^{c}$.

\section{F. Conclusion}

Under a natural requirement that the distribution of economic fluctuations does not depend on the choice of a monetary unit, we conclude that $t(x) \sim x^{\alpha}$ and thus,

$$
\rho(x) \sim \frac{\text { const }}{t(x)} \sim x^{-\alpha} .
$$

Thus, the power law is justified.

\section{Individual Stock: IdEALIZEd CASE}

A natural next question is: which value $\alpha$ should we expect? In other words, if we re-scale the monetary unit, what is the appropriate change in a time unit that leaves the dependence unchanged?

To answer this question, we will take into account a different relation between time and fluctuation size. Namely, until now, we considered fluctuations which occur within a single time quantum $\Delta t$. However, we can consider different time quanta: e.g., a time quantum $\Delta t^{\prime}=k \cdot \Delta t$ for some integer $k$.

We have mentioned that price fluctuations are reasonably accurately described by a random walk. In the random walk, fluctuations occurring at different time quanta are independent random variables with 0 mean. Let $\sigma$ denote the standard deviation of a price over the time quantum $\Delta t$. Then, the corresponding variance is equal to $\sigma^{2}$.

The fluctuation over a new time quantum $\Delta t^{\prime}=k \cdot \Delta t$ can be obtained by adding the fluctuations over $k$ original time quanta, of size $\Delta t$. These fluctuations are independent random variables. It is known that when we add several independent random variables, their variances add up. Thus, the variance of the fluctuations over a new time quantum is equal to the sum of $k$ terms equal to $\sigma^{2}$, i.e., to $k \cdot \sigma^{2}$. Hence, the mean square fluctuation is equal to $\sqrt{k} \cdot \sigma$.

Thus, when the numerical value of the time increases by a factor of $k$, the corresponding (mean square) value of the fluctuation increases by a factor of $\sqrt{k}$.

In terms of the coefficients $\lambda$ and $r(\lambda)$, this means that when $\lambda=\sqrt{k}$, we have $r(\lambda)=k$, i.e.,

$$
r(\lambda)=\lambda^{2} .
$$

For $t(x)=C \cdot x^{\alpha}$, the scale-invariance requirement (13) implies that

$$
r(\lambda)=\frac{t(\lambda \cdot x)}{t(x)}=\frac{C \cdot(\lambda \cdot x)^{\alpha}}{C \cdot x^{\alpha}}=\lambda^{\alpha} .
$$


By comparing (19) and (20), we conclude that $\lambda=2$. Thus, due to the formula (18), we conclude that in the idealized situation, for an individual stock, the probability density function has the form

$$
\rho(x) \sim x^{-2},
$$

and, thus,

$$
P\left(x>x_{0}\right) \sim x^{-1} .
$$

\section{FROM THE IdEALIZED CASE OF AN INDIVIDUAL} StOCK TO STOCK MARKET: TwO APPROACHES

In the previous section, we considered an idealized case of an individual stock which is not interacting with other stock prices. In reality, stocks are inter-related: a change in one stock price causes a change in prices of other stocks. How can we take this dependence into account?

In this paper, we describe two approaches for taking this dependence into account:

- a probabilistic approach, and

- a fuzzy approach.

We will show that both approaches lead to the same distribution - which makes us even more confident that this is indeed a correct distribution.

\section{FROM THE IDEALIZED CASE OF AN INDIVIDUAL STOCK TO STOCK MARKET: Probabilistic ApProACH}

Stocks are usually classified based on four characteristics:

- the size of the company (large cap vs. mid cap vs. small cap stocks);

- the size of the dividend (income stocks vs. non-income ones);

- cyclicity (cyclic stocks vs. defensive stocks);

- stability (less risky value stocks vs. more aggressive and more risky growth stocks).

Crudely speaking, this means that we have 4 different extreme types of stocks, and every stock is, in some reasonable sense, equivalent to a combination of these different 4 types.

It is known that different types of stocks behave differently and reasonably independently from each other - this fact is used to justify the need for a balanced combination of different types of stocks in an investment portfolio.

In a stock market, usually, individual stock price changes largely compensate each other, so the fluctuations of the stock market index are much smaller than the fluctuations individual stock prices. For a stock market index to really change, the majority of stocks must experience the corresponding drastic change.

Since we reason about four types of stock, "the majority" means that at least three types of stock out of four must experience a drastic fluctuation. The probability that each type of stock experiences a fluctuation of size $x_{0}$ is proportional to $x_{0}^{-1}$. Since stocks of different type behave independently, the probability that stocks of 3 types experience the same size fluctuation is equal to the product of the three values $x_{0}^{-1}$, i.e., to

$$
P\left(x>x_{0}\right) \sim x_{0}^{-1} \cdot x_{0}^{-1} \cdot x_{0}^{-1} \sim x_{0}^{-3} .
$$

Thus, we have indeed justified the cubic dependence (7).

\section{From the IdEALIZED CASE OF AN INDIVIDUAL StOCK TO STOCK MARKET: FUZZY APPROACH}

For large (but reasonable size) fluctuations, we can use the individual stock description, with the probability density function (21): $\rho(x) \sim x^{-2}$. Correspondingly, a reasonable membership function $\mu(x)$ can be obtained by normalizing this expression:

$$
\mu_{\text {large }}(x)=\frac{\rho(x)}{\max _{y} \rho(y)} \sim x^{-2} .
$$

We are interested in very large (crisis-type) fluctuations. In fuzzy logic, the most widely used way to go

- from the membership function of a property such as "large"

- to the membership function corresponding to the hedged property "very large"

is to take the square of the original membership function (see, e.g., [8], [14]):

$$
\mu_{\text {very_large }}(x)=\mu_{\text {large }}^{2}(x) \sim\left(x^{-2}\right)^{2}=x^{-4} .
$$

Thus, the corresponding probability density function is also proportional to $x^{-4}$. So, we have indeed justified the formula (6).

Comment. We have justified the same empirical distribution by using two different approaches:

- the probabilistic approach and

- the fuzzy approach.

By their very origins,

- the probabilistic approach is usually based on the (more) mathematical analysis, while

- the fuzzy approach is more oriented towards natural language and commonsense reasoning.

By comparing the two justifications, one can see that, in perfect accordance with this difference, the derivation is much clearer (and easier) when we use fuzzy logic.

\section{CONCLUSION}

To enhance the use of accurate empirical descriptions of economic fluctuations, it is necessary to provide a theoretical justification for these empirical descriptions. In this paper, we provided two such justifications, based on probabilistic and fuzzy approaches. The fact that both justifications lead to the same distribution further increases our confidence in this empirical distribution.

\section{ACKNOWLEDGMENTS}

This work was supported in part by NSF grant HRD0734825 and by Grant 1 T36 GM078000-01 from the National Institutes of Health.

The authors are thankful to the anonymous referees for valuable suggestions. 


\section{REFERENCES}

[1] J. Aczel, Lectures on Functional Equations and Their Applications, Dover Publ., New York, 2006.

[2] L. Bachelier, Théorie de la spéculation, PhD Dissertation, l'Ecole Normal Supérieure, Paris, 1900.

[3] B. K. Chakrabarti, A. Chakraborti, and A. Chatterjee, Econophysics and Sociophysics: Trends and Perspectives, Wiley-VCH, Berlin, 2006.

[4] A. Chatterjee, S. Yarlagadda, B. K. Chakrabarti, Econophysics of Wealth Distributions, Springer-Verlag Italia, Milan, 2005

[5] J. D. Farmer and T. Lux (eds.), Applications of statistical physics in economics and finance, a special issue of the Journal of Economic Dynamics and Control, 2008, Vol. 32, No. 1, pp. 1-320.

[6] X. Gabaix, G. Parameswaran, P. Vasiliki, and H. E. Stanley, "Understanding the cubic and half-cubic laws of financial fluctuations", Physica A, 2003, Vol. 324, pp. 1-5.

[7] X. Gabaix, G. Parameswaran, P. Vasiliki, and H. E. Stanley, "A theory of power-law distributions in financial market fluctuations", Nature, 2003, Vol. 423, No. 6937, pp. 267-270.

[8] G. Klir and B. Yuan, Fuzzy sets and fuzzy logic: theory and applications. Prentice Hall, Upper Saddle River, New Jersey, 1995.

[9] B. Mandelbrot, "The variation of certain speculative prices", J. Business, 1963, Vol. 36, pp. 394-419.

[10] B. Mandelbrot and R. L. Hudson, The (Mis)behavior of Markets: A Fractal View of Financial Turbulence, Basic Books, 2006.
[11] R. N. Mantegna and H. E. Stanley, An Introduction to Econophysics: Correlations and Complexity in Finance, Cambridge University Press, Cambridge, Massachusetts, 1999.

[12] J. McCauley, Dynamics of Markets, Econophysics and Finance, Cambridge University Press, Cambridge, Massachusetts, 2004.

[13] H. T. Nguyen and V. Kreinovich, Applications of continuous mathematics to computer science, Kluwer, Dordrecht, 1997.

[14] H. T. Nguyen and E. A. Walker, A first course in fuzzy logic, CRC Press, Boca Raton, Florida, 2005.

[15] J. Pexider, Notiz uber Funktionaltheoreme, Monatsch. Math. Phys., 1903, Vol. 14, pp. 293-301.

[16] B. Roehner, Patterns of Speculation - A Study in Observational Econophysics, Cambridge University Press, Cambridge, Massachusetts, 2002.

[17] D. J. Sheskin, Handbook of Parametric and Nonparametric Statistical Procedures, Chapman \& Hall/CRC Press, Boca Raton, Florida, 2007.

[18] H. E. Stanley, "Econophysics and the current economic turmoil", American Physical Society News, 2008, Vol. 17, No. 11, p. 8.

[19] H. E. Stanley, L. A. N. Amaral, P. Gopikrishnan, and V. Plerou, "Scale invariance and universality of economic fluctuations", Physica A, 2000, Vol. 283, pp. 31-41.

[20] P. Vasiliki and H. E. Stanley, "Stock return distributions: tests of scaling and universality from three distinct stock markets", Physical Review E: Statistical, Nonlinear, and Soft Matter Physics, 2008, Vol. 77, No. 3, Pt. 2, Publ. 037101. 